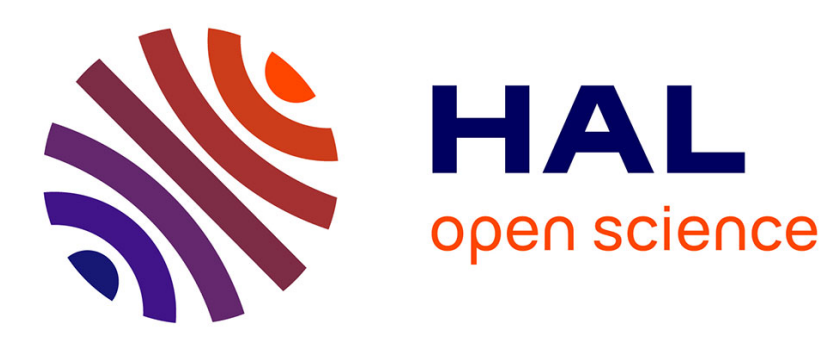

\title{
Perfectly Matched Layers for the heat and advection-diffusion equations
}

\author{
Nicolas Lantos, Frédéric Nataf
}

\section{To cite this version:}

Nicolas Lantos, Frédéric Nataf. Perfectly Matched Layers for the heat and advection-diffusion equations. 2010. hal-00454089

\author{
HAL Id: hal-00454089 \\ https://hal.science/hal-00454089
}

Preprint submitted on 23 Feb 2010

HAL is a multi-disciplinary open access archive for the deposit and dissemination of scientific research documents, whether they are published or not. The documents may come from teaching and research institutions in France or abroad, or from public or private research centers.
L'archive ouverte pluridisciplinaire HAL, est destinée au dépôt et à la diffusion de documents scientifiques de niveau recherche, publiés ou non, émanant des établissements d'enseignement et de recherche français ou étrangers, des laboratoires publics ou privés. 


\title{
Perfectly Matched Layers for the heat and advection-diffusion equations
}

\author{
Nicolas Lantos (Natixis Corporate Solutions bank and UPMC), \\ Frédéric Nataf (LJLL, University of Paris VI) ${ }^{\dagger}$
}

February 22, 2010

\begin{abstract}
We design a perfectly matched layer for the advection-diffusion equation. We show that the reflection coefficient is exponentially small with respect to the damping parameter and the width of the PML and this independently of the advection and of the viscosity. Numerical tests assess the efficiency of the approach.
\end{abstract}

\section{Introduction}

We are concerned here with the problem of truncating domains to compute numerical solutions of problems in unbounded domains so that the solution of the problem in the reduced domain is a good approximation to the solution of the original problem. In their seminal work on the wave equation, Engquist and Majda [BA77] introduced a quite general technique to address this problem by designing absorbing boundary conditions (ABC). Their technique has been applied to various equations and systems of equations in many fields: acoustics, electromagnetism, fluid dynamics elastodynamics and so on. As far as the heat equation is concerned, in [Jol89], [Giv89], [LH95] and [Dub96], ABCs are designed at the continuous level and in [EM06] at the discrete level. In all these works, the difficulty lies in the approximation of the square root of a partial differential operator by a partial differential operator. This problem is inherent to the application of the procedure in [BA77] to the heat operator. Let us mention also the use of analytic solution with fast Fourier transforms, see [LG07] and references therein.

For hyperbolic equations such as the wave or Maxwell equations, a different way to handle artificial boundaries was introduced by Berenger [Ber94] and [Ber96]. In this method, the computational domain is surrounded by a

\footnotetext{
*lantos@ann.jussieu.fr

${ }^{\dagger}$ nataf@ann.jussieu.fr
} 
dissipative and non reflexive artificial media (perfectly matched layer, PML). There is no need to approximate the square root of an operator by a partial differential operator.

Since then, many works have been devoted to a better understanding of their principle and behavior see [MPV98], [ZC96], [CW94], [LS00] [MC98] [BFJ03][BJ02] [AGH02] to extensions to other geometries, see [ST04] [CM98], or equations see [HN02] [AGH99][DJ03] [Nat06] and [BDM10]. In these works, the equations are hyperbolic and the need for a PML comes from the propagative modes that exist in the solution. For propagative equations, the purpose of a PML is to turn a propagative mode into a vanishing one.

In this paper, we consider a parabolic equation for which there are only vanishing modes. In this paper, we show that it is nevertheless possible to design and test a PML for the advection-diffusion equation:

$$
\mathcal{L}(u):=\frac{\partial u}{\partial t}+a \frac{\partial u}{\partial x}+b \frac{\partial u}{\partial y}-\nu \Delta u
$$

including the heat equation as a special case. In our work, the purpose of the PML is to turn a slowly vanishing mode into a rapidly vanishing one. The efficiency is not dependent on the parameters of the equation such as the viscosity or the convection, see equation (8).

The paper is organized as follows. In the first part, we analyze the operator (1) in the Fourier space and imantroduce the perfectly matched layers to the advection-diffusion equation. In the second part, we apply this method to numerical computation to validate our approach.

\section{Perfectly Matched Layers}

\section{$1.1 \quad$ Fourier analysis of the operator $\mathcal{L}$}

In order to study the operator $\mathcal{L}$, we look for solutions of the equation $\mathcal{L}(u)=0$ and make use of the Fourier transform. Let $u(t, x, y)$ be a function and $\hat{\hat{u}}(\omega, x, k)$ be its Fourier transform w.r.t. the variables $t$ and $y$ and let $\mathcal{F}^{-1}$ denote the inverse Fourier transform. We have:

$$
\left(i \omega+a \partial_{x}+b i k-\nu \partial_{x x}+\nu k^{2}\right)(\hat{\hat{u}}(\omega, x, k))=0 .
$$

For fixed $\omega$ and $k$, this is an ordinary differential equation in the variable $x$ whose solutions are of the form

$$
\hat{\hat{u}}(\omega, x, k)=\alpha(\omega, k) \exp \left(\lambda^{+}(\omega, k) x\right)+\beta(\omega, k) \exp \left(\lambda^{-}(\omega, k) x\right)
$$


where

$$
\lambda^{ \pm}(\omega, k):=\frac{\frac{a}{\nu} \pm \sqrt{\frac{a^{2}}{\nu^{2}}+\frac{4}{\nu}\left(i \omega+i k b+\nu k^{2}\right)}}{2}
$$

and $\alpha$ and $\beta$ are fixed by the boundary conditions.

\subsection{Definition of the PML equations}

The operator $\mathcal{L}$ (see eq. (1) is originally defined in the whole plane $\mathbb{R}^{2}$ and we want to truncate the domain $x>0$ by a PML. The PML model for this operator $\mathcal{L}$ is defined by replacing the $x$-derivative by a "pml" $x$-derivative. The definition is as follows. Let $\sigma>0$ be a positive damping parameter, we define

$$
\partial_{x}^{p m l}(u):=\mathcal{F}^{-1}\left(\frac{i \omega+i k b}{i \omega+i k b+\frac{\nu}{4} \sigma} \partial_{x} \hat{\hat{u}}(\omega, x, k)\right)
$$

and

$$
\mathcal{L}_{p m l}:=\partial_{t}+a \partial_{x}^{p m l}+b \partial_{y}-\nu\left(\partial_{x}^{p m l}\right)^{2}-\nu \partial_{y y}
$$

be the PML equation with the following interface conditions at $\mathrm{x}=0$ between the solution $u_{c d}$ in the convection-diffusion media and $u_{p m l}$ the solution in the PML media:

$$
u_{c d}=u_{p m l} \text { and } \partial_{x}\left(u_{c d}\right)=\partial_{x}^{p m l}\left(u_{p m l}\right)
$$

\subsection{Reflection coefficient}

We show in this section that the reflection coefficient for a PML of width $\delta>0$ is exponentially small with respect to the damping parameter $\sigma$ and the width $\delta$ and this independently of the advection $(a, b)$ and of the viscosity $\nu$, see formula (8). For this, we use the following setting which mimics the classical computation of the reflection coefficient for PML for the wave equation. The function

$$
u_{i n c}:=\mathcal{F}^{-1}\left(\exp \left(\lambda^{-}(\omega, k) x\right)\right)
$$

satisfies

$$
\mathcal{L}\left(u_{\text {inc }}\right)=0
$$

and $u_{i n c}$ tends to zero as $x$ tends to infinity. We approach this special solution by the following problem where the domain is truncated on the right by the PML:

Find $\left(u_{c d}, u_{p m l}\right)$ such that

$$
\begin{aligned}
& \mathcal{L}\left(u_{c d}\right)=0, t>0, x<0, y \in \mathbf{R} \\
& \mathcal{L}_{p m l}\left(u_{p m l}\right)=0, t>0, \delta>x>0, y \in \mathbf{R} \\
& u_{p m l}(t, \delta, y)=0, t>0, y \in \mathbf{R} \\
& u_{c d}-u_{\text {inc }} \text { tends to } 0 \text { as } x \text { to }-\infty,
\end{aligned}
$$

and the interface conditions (5) are satisfied. 
We take the Fourier transform of the above system and we get:

$\hat{\hat{\mathcal{L}}}\left(\hat{\hat{u}}_{c d}\right)=0, t>0, x<0, y \in \mathbf{R}$

$\hat{\hat{\mathcal{L}}}_{p m l}\left(\hat{\hat{u}}_{p m l}\right)=0, t>0, \delta>x>0, y \in \mathbf{R}$

$\hat{\hat{u}}_{p m l}(t, \delta, y)=0, t>0, y \in \mathbf{R}$

$\hat{\hat{u}}_{c d}-\exp \left(\lambda^{-}(\omega, k) x\right)$ tends to 0 as $x$ to $-\infty$,

and the Fourier transform of the interface conditions (5) are satisfied at $x=0$.

Easy computations show that $u_{c d}$ and $u_{p m l}$ have the following expression with $R, \alpha$ and $\beta$ coefficients that will be determined in the sequel:

$$
u_{c d}:=\exp \left(\lambda^{-} x\right)+R \exp \left(\lambda^{+} x\right)
$$

and

$$
u_{p m l}:=\alpha \exp \left(\lambda_{p m l}^{-} x\right)+\beta \exp \left(\lambda_{p m l}^{+} x\right) .
$$

where

$$
\lambda_{p m l}^{ \pm}:=\frac{i \omega+i k b+\frac{\nu}{4} \sigma}{i \omega+i k b} \lambda^{ \pm}
$$

If $R$ were equal to zero, the solution in the left-plane would be equal to $u_{\text {inc }}$ and the PML would be an exact way to truncate the computational domain. Thus, the smallness of $R$ is a measure of the quality of the PML procedure and defines a reflection coefficient.

By using (6), the Fourier transform of the interface conditions (5) at $x=0$ yield:

$$
1+R=\alpha+\beta \text { and } \lambda^{-}+R \lambda^{+}=\alpha \lambda^{-}+\beta \lambda^{+}
$$

The Dirichlet boundary condition at the end of the PML $(x=\delta)$ gives:

$$
\alpha \exp \left(\lambda_{p m l}^{-} \delta\right)+\beta \exp \left(\lambda_{p m l}^{+} \delta\right)=0 .
$$

A simple calculation yields the formula for the convergence rate:

$$
R=-\exp \left(-\left(\lambda_{p m l}^{+}-\lambda_{p m l}^{-}\right) \delta\right)
$$

We prove now that we have a uniform bound on the reflection coefficient $R$ independently of the physical parameters $(a, b, \nu)$ and of the Fourier variables $(\omega, k)$ :

$$
|R| \leq \exp (-\sqrt{2 \sigma} \delta)
$$

Proof: We need to bound the real part of $\lambda_{p m l}^{+}-\lambda_{p m l}^{-}$from below by $\sqrt{2 \sigma}$. We have

$$
\lambda_{p m l}^{+}-\lambda_{p m l}^{-}=\left(1+\frac{\sigma \nu}{4(i \omega+i k b)}\right) \sqrt{\frac{a^{2}}{\nu^{2}}+\frac{4}{\nu}\left(i \omega+i k b+\nu k^{2}\right)} .
$$


Let $p$ and $q$ be two real numbers such that:

$$
p+i q:=\sqrt{\frac{a^{2}}{\nu^{2}}+\frac{4}{\nu}\left(i \omega+i k b+\nu k^{2}\right)}
$$

Let us define

$$
c:=\frac{a^{2}}{\nu^{2}}+4 k^{2} \text { and } d:=\frac{4}{\nu}(\omega+k b) .
$$

Without loss of generality, assume now that $d<0$. We have:

$$
p=\frac{1}{2} \sqrt{2 \sqrt{c^{2}+d^{2}}+2 c} \text { and } q=-\frac{1}{2} \sqrt{2 \sqrt{c^{2}+d^{2}}-2 c}
$$

With these notations,

$$
\begin{aligned}
\operatorname{Re}\left(\lambda_{p m l}^{+}-\lambda_{p m l}^{-}\right) & =p+\frac{\sigma q}{d} \\
& =\frac{1}{\sqrt{2}}\left(\sqrt{\sqrt{c^{2}+d^{2}}+c}-\frac{\sigma}{d} \sqrt{\sqrt{c^{2}+d^{2}}-c}\right) \\
& =\frac{1}{\sqrt{2}}\left(\sqrt{\sqrt{c^{2}+d^{2}}+c}+\sigma \frac{1}{\sqrt{\sqrt{c^{2}+d^{2}}+c}}\right)
\end{aligned}
$$

The function $x \mapsto x+\sigma / x$ has a minimum value of $2 \sqrt{\sigma}$ reached at $x=\sqrt{\sigma}$. Thus,

$$
\operatorname{Re}\left(\lambda_{p m l}^{+}-\lambda_{p m l}^{-}\right) \geq \sqrt{2 \sigma} .
$$

The proof is similar for $d>0$.

It is easy to check that the same reflection coefficient is obtained if the PML is used to truncate the computational domain on the left.

A PML- $y$ used for truncating the domain in the $y$ direction would consist in replacing in the operator $\mathcal{L}$ the $y$ derivatives by a "pml" derivative in the $y$ direction defined as follows ( $\xi$ is the dual variable of $x$ for the Fourier transform in the $x$ direction):

$$
\partial_{y}^{p m l}(u):=\mathcal{F}_{t, x}^{-1}\left(\frac{i \omega+i \xi a}{i \omega+i \xi a+\frac{\nu}{4} \sigma} \partial_{x} \hat{\hat{u}}(\omega, \xi, y)\right)
$$

The PML- $y$ equations of the convection-diffusion operator $\mathcal{L}$ for the truncation of the domain in the $y$ direction reads:

$$
\mathcal{L}_{p m l, y}:=\partial_{t}+a \partial_{x}+b \partial_{y}^{p m l}-\nu\left(\partial_{x}\right)^{2}-\nu \partial_{y y}^{p m l}
$$

In order to give a complete definition of a PML bordering a rectangular computational domain, we have three possibilities for the corner region. The first one consists in designing a third PML model in the corner that 
is compatible with both PML- $x$ and PML- $y$ as was done for the Maxwell system in [Ber94] for instance. The second possibility is to use prismatoidal coordinates [LS01]. The advantage is that it allows for arbitrary convex computational domains and not only rectangular ones. The third possibility consists in not designing a new PML for the corner but simply in placing side by side PML- $x$ and PML- $y$ regions. We have implemented this last simple approach where convection coefficients $a$ and $b$ are chosen null in the PML' changes of variable (3) and (9) and we obtain good results for mild convection term, see $\S 2$. Of course, the two first options deserve further investigations.

\section{$2 \quad$ Numerical Results}

We present in this section the numerical application of the method defined in the previous section. We will first introduce the numerical experiment and study the sensitivity of the computation with respect to the profile of the damping parameter. We will then present the numerical results obtained for the heat equation with or without advection terms and compare it with the classical Neumann or Dirichlet homogeneous boundary conditions.

\section{$2.1 \quad$ Numerical experiment}

We are interesting in solving this advection-diffusion problem:

$$
\left\{\begin{array}{l}
\frac{\partial u}{\partial t}+a \frac{\partial u}{\partial x}+b \frac{\partial u}{\partial y}-\nu \Delta u=0 \quad \text { with }(x, y) \in \mathbb{R}^{2}, 0 \leq t \leq T \\
u(x, y, 0)=u_{0}(x, y)
\end{array}\right.
$$

where $u_{0}$ is the initial condition, $\nu$ is the viscosity, $(a, b)$ is the velocity field and $T$ is the final time.

To carry out numerical applications, we choose to solve this problem in the particular case where an analytical solution is known. This will simplify the study of the accuracy of the numerical applications. Problem (11) with a Gaussian density (12) as initial condition :

$$
u_{0}(x, y)=\frac{1}{\gamma} e^{-\frac{x^{2}+y^{2}}{\nu \gamma}}
$$

admits the analytical solution $u^{E x}$ :

$$
u^{E x}(x, y, t)=\frac{1}{4 t+\gamma} e^{-\frac{(x+a t)^{2}+(y+b t)^{2}}{\nu(4 t+\gamma)}} .
$$

We approximate our problem with a P1-triangular Finite Element Method $(\mathrm{FEM})$ on a truncated domain $\Omega_{L}=[-L \times s t d v,+L \times s t d v]^{2}$ where $s t d v=$ 
$\sqrt{2 \nu T}$ is the standard deviation merging the viscosity $(\nu)$ and the final time $(T)$ and $L$ is a positive parameter. The associated approximated solution of the FEM is denoted as $u_{h}$, with $h$ stands for the space step. We discretize the time derivative with a implicit Euler scheme.

To study the numerical results, we define with a parameter $0<\eta<L$ an arbitrary domain $\Omega_{\eta}=[-\eta \times s t d v,+\eta \times s t d v]^{2}$ nested in $\Omega_{L}$ where errors will be computed. We also introduce $u_{h}^{\infty}$ a reference numerical solution of (11) on a computational domain $\Omega_{L}$ sufficiently large to avoid any boundary conditions issue. Finally, let $\epsilon_{h}^{\infty}(x, y, t)=\left|u_{h}(x, y, t)-u_{h}^{\infty}(x, y, t)\right|$ and $\epsilon^{E x}(x, y, t)=\left|u_{h}(x, y, t)-u^{E x}(x, y, t)\right|$ be two absolute errors expressed in percentage. The $\epsilon^{E x}$ error is composed by a twofold error: the discretization error of the FEM and the error introduced by the truncation of the domain. On the other hand, we introduced the error $\epsilon_{h}^{\infty}$ to only highlight the truncation error.

\subsection{Setup of the damping parameters for the heat equation}

In actual numerical simulations, due to the discretization of the PML equations and to the finite width of the PML, small reflections occur. The damping parameter $\sigma$ has to be null at the interface of medias in order to control the numerical reflection introduced at the discrete level. This is why the damping parameter $\sigma$ has the following profile $\sigma(z)=\alpha\left(\frac{z}{\delta}\right)^{\beta}$ where $z$ is the distance to the interface in the normal direction to the interface $(z \in[0, \delta])$ and $(\alpha, \beta)$ are arbitrary constants that will be chosen in the following section. This was the original choice introduced by Berenger ([Ber94]). We study the sensitivity of the computation with respect to the damping parameters. We took the following parameters for the discretization scheme $L=1$ and the number of discretization nodes in the PML is 11 . The viscosity $\nu$ is 0.5 and the final time $T=5$. The initial condition (12) is chosen with $\gamma=0.2$. We solve $u_{h}^{\infty}$ on $\Omega_{L}$, with $L=5$ and errors in the $L^{2}$ and $L^{\infty}$ norm are always computed on $\Omega_{\eta}$ with $\eta=0.8$. The time step for all the numerical computations is $\Delta t=0.025$.

In FIG. 1, we plot $\left\|\epsilon_{h}^{\infty}\right\|_{\infty}$ as a function of $\alpha$ and $\beta$. After some strong variation, the error seems to stabilize at a value of $\approx 0.01 \%$ for $(\alpha, \beta) \in[20,100] \times[2,10]$. More precisely some small variations can be seen is this range, but without any change of magnitude. A further study may be deserved to adaptively optimize the profile to reduce the numerical reflection as it is done in [LU06].

\subsection{Comparison with classical boundary conditions}

We now present some numerical results obtained with the PML according to the setup parameters found in the previous section. To highlight the accuracy of the PML method we will compare our numerical application to 


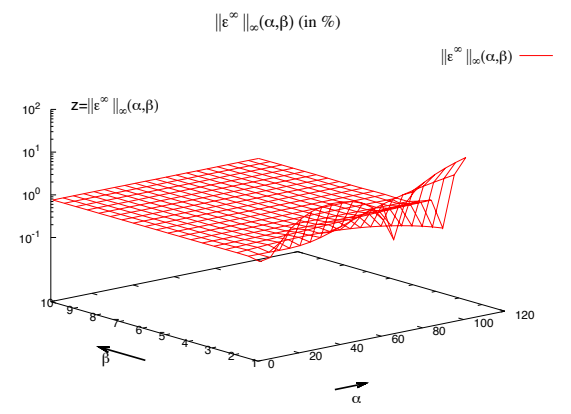

Figure 1: $\left\|\epsilon_{h}^{\infty}\right\|_{\infty}(\alpha, \beta)$ for a range of value: $\alpha \in[1,101], \beta \in[1,10]$

the more classical boundary conditions: Dirichlet or Neumann homogeneous. In order to have a fair comparison, when Neumann or Dirichlet boundary conditions are used the computational domain includes the PML zone. In figures $(2,3,4,5)$, the meshed surface of the curves in the middle represents $\Omega_{\eta}$ the domain where the error is computed, the dotted part stands for the rest of the physical domain $\left(\Omega_{L} \backslash \Omega_{\eta}\right)$.

\section{Heat equation}

The results are first presented for a pure heat equation with $\nu=0.5$. The parameters of the discretization scheme are the following: $L$ is $1, \eta$ is 0.8 , 
$h$ is the ratio $2 s t d v / 51$. The parameters for the PML media are $\alpha=10$, $\beta=2$ and the number of mesh point in the PML is $N=11$. The space step remains constant between the physical and the PML domain.

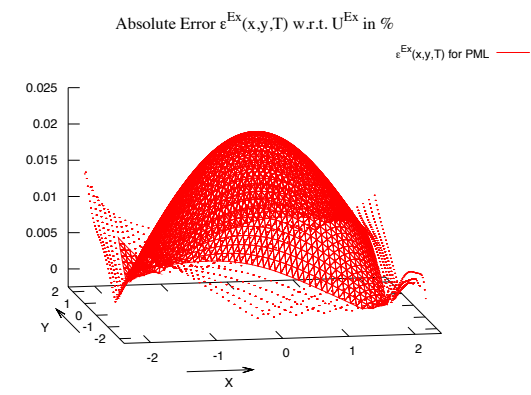

Figure 2: The error $\epsilon^{E x}(x, y, T)$ w.r.t. $U^{E x}$ (in \%) for the PML.

The numerical results are shown in Fig. 2 and Fig. 3 and figures are presented in table 1 for various boundary conditions. The table shows that for Dirichlet and Neumann boundary conditions the error w.r.t. the exact solution comes mainly from the truncation error and not from the discretization of the equation.

We see on Fig. 2 that the most important part of the error, when using the PML, is in the center of the computational domain far from the artificial 


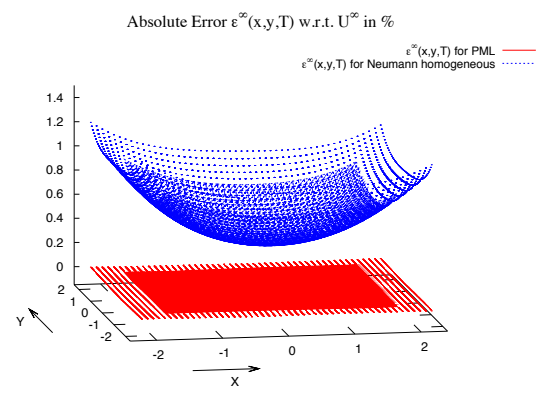

Figure 3: The error $\epsilon_{h}^{\infty}(x, y, T)$ w.r.t. $U^{\infty}$ (in \%) for Neumann homogeneous boundary condition and the PML. The Dirichlet boundary condition results are roughly the same as Neumann.

boundary. The PML scheme thus capture efficiently the solution at the boundary of the physical domain and the remaining error only comes from the discretization scheme. As shown in Fig. 3 the PML outperform the other classical boundary conditions.

\section{Advection-diffusion equation}

We now assume a non null advection coefficient in the $x$-direction $(a=0.5$ and $b=0)$ with the same viscosity. For larger velocity coefficients, we are 


\begin{tabular}{|c|c|c|c|c|c|c|}
\hline & $\epsilon_{h}^{\infty}(0,0)$ & $\left\|\epsilon_{h}^{\infty}\right\|_{2}$ & $\left\|\epsilon_{h}^{\infty}\right\|_{\infty}$ & $\epsilon^{E x}(0,0)$ & $\left\|\epsilon^{E x}\right\|_{2}$ & $\left\|\epsilon^{E x}\right\|_{\infty}$ \\
\hline$u_{h}^{\infty}$ & 0 & 0 & 0 & $0.0263 \%$ & $0.0154 \%$ & $0.0263 \%$ \\
\hline$u_{h}^{N}$ & $0.3533 \%$ & $0.5915 \%$ & $1.0194 \%$ & $0.3795 \%$ & $0.6038 \%$ & $1.0188 \%$ \\
\hline$u_{h}^{D}$ & $0.3139 \%$ & $0.5363 \%$ & $0.8545 \%$ & $0.3139 \%$ & $0.5363 \%$ & $0.8545 \%$ \\
\hline$u_{h}^{P M L}$ & $0.0042 \%$ & $0.0030 \%$ & $0.0075 \%$ & $0.0221 \%$ & $0.0131 \%$ & $0.0221 \%$ \\
\hline
\end{tabular}

Table 1: Numerical results obtained for the heat equation for various boundary conditions: Neumann homogeneous (N), Dirichlet homogeneous (D), and Perfectly Matched Layers (PML)

going to deal with an advection dominated equation and a simple Neumann boundary condition will surely have a better efficiency. The PML change of variable (see (3) for example) introduces a convection dominated problem in the associated partial differential equations system. We use SUPG to improve the resolution.

The parameters of the discretization scheme remain the same as before and the numerical results are shown in Fig. 4 and Fig. 5 and in table 2 for various boundary conditions.

\begin{tabular}{|c|c|c|c|c|c|c|}
\hline & $\epsilon_{h}^{\infty}(0,0)$ & $\left\|\epsilon_{h}^{\infty}\right\|_{2}$ & $\left\|\epsilon_{h}^{\infty}\right\|_{\infty}$ & $\epsilon^{E x}(0,0)$ & $\left\|\epsilon^{E x}\right\|_{2}$ & $\left\|\epsilon^{E x}\right\|_{\infty}$ \\
\hline$u_{h}^{\infty}$ & 0 & 0 & 0 & $0.0264 \%$ & $0.0199 \%$ & $0.0340 \%$ \\
\hline$u_{h}^{N}$ & $0.2066 \%$ & $0.3467 \%$ & $0.8491 \%$ & $0.2330 \%$ & $0.3636 \%$ & $0.8564 \%$ \\
\hline$u_{h}^{D}$ & $0.1546 \%$ & $0.3809 \%$ & $1.0785 \%$ & $0.1546 \%$ & $0.3809 \%$ & $1.0785 \%$ \\
\hline$u_{h}^{P M L}$ & $0.0019 \%$ & $0.0035 \%$ & $0.0129 \%$ & $0.0245 \%$ & $0.0173 \%$ & $0.0288 \%$ \\
\hline
\end{tabular}

Table 2: Numerical results obtained under a advection-diffusion model ( $a=$ $0.5, b=0)$ for distinct boundary condition: Neumann homogeneous $(\mathrm{N})$, Dirichlet homogeneous (D), and Perfectly Matched Layers (PML)

Once again, we notice, see Fig 4, that the error is bigger inside the physical domain away from the boundary. This error thus only come from the discretization scheme and the PML scheme enables to efficiently treat advection-diffusion equation. From Table 2, we see that for Dirichlet and Neumann boundary conditions, the error is very strongly dominated by the truncation error.

We finally introduce a third example with non null advection coefficient in both directions $(a=b=0.25)$. The numerical results are shown in Fig. 6 and Fig. 7 and in table 3 for several boundary conditions in term of various error. We observe the same order of accuracy as before in the center of the computational domain except as shown in Fig. 6 in the corners. This is due to our arbitrary choice of the treatment of the corner as explained before at the end of section 1.

The numerical scheme proved to be stable for long time computations in all cases. 
Absolute Error $\varepsilon^{\mathrm{Ex}}(\mathrm{x}, \mathrm{y}, \mathrm{T})$ w.r.t. $\mathrm{U}^{\mathrm{Ex}}$ in \%

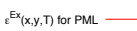

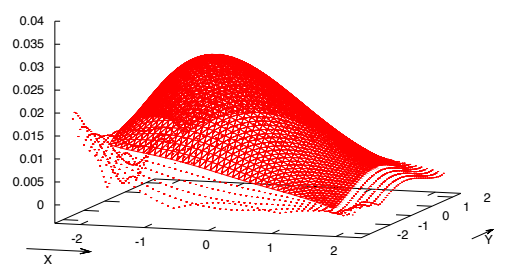

Figure 4: The error $\epsilon^{E x}(x, y, T)$ w.r.t. $U^{E x}$ (in \%) for the PML boundary condition with positive advection coefficient in the $x$-direction.

\section{Conclusion \& Perspectives}

In this article, we designed a perfectly matched layer for the heat and/or advection-diffusion equation. After its definition, we prove that the reflection coefficient is exponentially small with respect to the damping parameter and the width of the PML. It is worth noticing that the reflection coefficient is independent of the equation parameters such as velocity or viscosity. We have implemented this method with a P1-finite element method and its efficiency is highlighted by numerical results. 


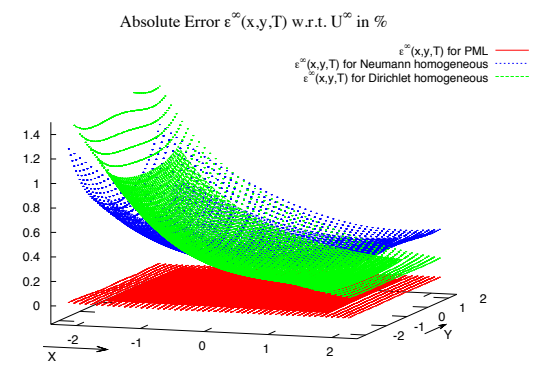

Figure 5: The error $\epsilon_{h}^{\infty}(x, y, T)$ w.r.t. $U^{\infty}$ (in \%) for Neumann homogeneous, Dirichlet homogeneous and PML boundary condition.

\section{References}

[AGH99] S. Abarbanel, D. Gottlieb, and J. S. Hesthaven. Well-posed perfectly matched layers for advective acoustics. J. Comput. Phys., 154(2):266-283, 1999.

[AGH02] S. Abarbanel, D. Gottlieb, and J. S. Hesthaven. Long time behavior of the perfectly matched layer equations in computational electromagnetics. In Proceedings of the Fifth International Con- 


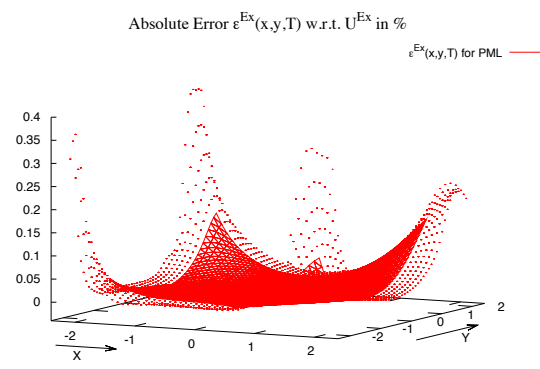

Figure 6: The error $\epsilon^{E x}(x, y, T)$ w.r.t. $U^{E x}$ (in \%) for the PML boundary condition with positive advection coefficient in the $x$-direction.

ference on Spectral and High Order Methods (ICOSAHOM-01) (Uppsala), volume 17, pages 405-422, 2002.

[BA77] B.Engquist and A.Majda. Absorbing boundary conditions for the numerical simulation of waves. Math. Comp, 31:629-651, 1977.

[BDM10] H Barucq, J Diaz, and Tlemcani M. New absorbing layers conditions for short water waves. J. Comput. Phys., 229:58-72, 2010.

[Ber94] J.P. Berenger. A perfectly matched layer for the absorption of electromagnetic waves. J. Comput. Phys., 114(2):185-200, 1994. 


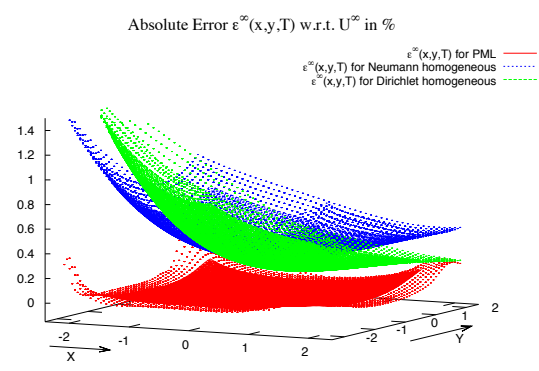

Figure 7: The error $\epsilon_{h}^{\infty}(x, y, T)$ w.r.t. $U^{\infty}$ (in \%) for Neumann homogeneous, Dirichlet homogeneous and PML boundary condition.

[Ber96] J. P. Berenger. Three-dimensional perfectly matched layer for the absorption of electromagnetic waves. J. Comput. Phys., 127(2):363-379, 1996.

[BFJ03] E. Bécache, S. Fauqueux, and P. Joly. Stability of perfectly matched layers, group velocities and anisotropic waves. J. Comput. Phys., 188(2):399-433, 2003.

[BJ02] Eliane Bécache and Patrick Joly. On the analysis of Bérenger's perfectly matched layers for Maxwell's equations. M2AN Math. 


\begin{tabular}{|c|c|c|c|c|c|c|}
\hline & $\epsilon_{h}^{\infty}(0,0)$ & $\left\|\epsilon_{h}^{\infty}\right\|_{2}$ & $\left\|\epsilon_{h}^{\infty}\right\|_{\infty}$ & $\epsilon^{E x}(0,0)$ & $\left\|\epsilon^{E x}\right\|_{2}$ & $\left\|\epsilon^{E x}\right\|_{\infty}$ \\
\hline$u_{h}^{\infty}$ & 0 & 0 & 0 & $0.0274 \%$ & $0.0182 \%$ & $0.0305 \%$ \\
\hline$u_{h}^{N}$ & $0.2634 \%$ & $0.4482 \%$ & $1.0619 \%$ & $0.2908 \%$ & $0.4633 \%$ & $1.0647 \%$ \\
\hline$u_{h}^{D}$ & $0.2205 \%$ & $0.4674 \%$ & $1.4788 \%$ & $0.2205 \%$ & $0.4674 \%$ & $1.4788 \%$ \\
\hline$u_{h}^{P M L}$ & $0.0094 \%$ & $0.0286 \%$ & $0.1464 \%$ & $0.0180 \%$ & $0.0261 \%$ & $0.1463 \%$ \\
\hline
\end{tabular}

Table 3: Numerical results obtained under a advection-diffusion model ( $a=$ $b=0.25)$ for distinct boundary condition: Neumann homogeneous $(\mathrm{N})$, Dirichlet homogeneous (D), and Perfectly Matched Layers (PML)

Model. Numer. Anal., 36(1):87-119, 2002.

[CM98] Francis Collino and Peter Monk. The perfectly matched layer in curvilinear coordinates. SIAM J. Sci. Comput., 19(6):2061-2090 (electronic), 1998.

[CW94] W. C. Chew and W. H. Weedon. A 3d perfectly matched medium from modified maxwell's equations with stretched coordinates. IEEE Trans. Microwave Opt. Technol. Lett., 7:599-604, 1994.

[DJ03] Julien Diaz and Patrick Joly. Stabilized perfectly matched layer for advective acoustics. In Mathematical and numerical aspects of wave propagation-WAVES 2003, pages 115-119. Springer, Berlin, 2003.

[Dub96] Eric Dubach. Artificial boundary conditions for diffusion equations: Numerical study. Journal of Computational and Applied Mathematics, 70:127-144, 1996.

[EM06] Matthias Ehrhardt and Ronald E. Mickens. Discrete artificial boundary conditions for the black-scholes equation of american options. Matheon preprint, 2006.

[Giv89] Numerical Methods in Thermal Problems, chapter Finite element analysis of heat problems in unbounded domains. Pineridge Press, Swansea, U.K., 1989.

[HN02] T. Hagstrom and I. Nazarov. Absorbing layers and radiation boundary conditions for jet flow simulations. In Proc. of the 8th AIAA/CEAS aeroacoustics conference, volume 60, 2002.

[Jol89] P. Joly. Pseudo-transparent boundary conditions for the diffusion equation. I. Math. Methods Appl. Sci., 11(6):725-758, 1989.

[LG07] Jing-Rebecca Li and Leslie Greengard. On the numerical solution of the heat equation. I. Fast solvers in free space. J. Comput. Phys., 226(2):1891-1901, 2007. 
[LH95] J. Rauch Laurence Halpern. Artificial boundary conditions for diffusion equations. Numerische Mathematik, 71:185-224, 1995.

[LS00] M. Lassas and E. Somersalo. On the existence and convergence of the solution of pml equations. Computing, 60:229-241, 2000.

[LS01] Matti Lassas and Erkki Somersalo. Analysis of the PML equations in general convex geometry. Proc. Roy. Soc. Edinburgh Sect. A, 131(5):1183-1207, 2001.

[LU06] Y.Y. LU. Minimizing the discrete reflectivity of perfectly matched layers. IEEE Photonics Technology Letters, 18(3):487-489, 2006.

[MC98] P. Monk and F. Collino. Optimizing the perfectly matched layer. In IUTAM Symposium on Computational Methods for Unbounded Domains (Boulder, CO, 1997), volume 49 of Fluid Mech. Appl., pages 245-254. Kluwer Acad. Publ., Dordrecht, 1998.

[MPV98] R. Mittra, Ü. Pekel, and J. Veihl. A theoretical and numerical study of Berenger's perfectly matched layer (PML) concept for mesh truncation in time and frequency domains. In Approximations and numerical methods for the solution of Maxwell's equations (Oxford, 1995), volume 65 of Inst. Math. Appl. Conf. Ser. New Ser., pages 1-19. Oxford Univ. Press, New York, 1998.

[Nat06] Frédéric Nataf. A new approach to perfectly matched layers for the linearized Euler system. J. Comput. Phys., 214(2):757-772, 2006 .

[ST04] I. Singer and E. Turkel. A perfectly matched layer for the Helmholtz equation in a semi-infinite strip. J. Comput. Phys., 201(2):439-465, 2004.

[ZC96] L. Zhao and A. C. Cangellaris. GT-PML: Generalized theory of perfectly matched layers and its application to the reflectionless truncation of finite-difference time-domain grids. IEEE Trans. Microwave Theory Tech, 44:2555-2563, 1996. 